\title{
Generalised Hamiltonian embedding of the Proca model
}

\author{
N. Banerjee and R. Banerjee \\ S. N. Bose National Centre for Basic Sciences \\ DB 17, Sector I, SaltLake, Calcutta 700064, India.
}

\begin{abstract}
We convert the second class Proca model into a first class theory by using the generalised prescription of Batalin, Fradkin and Tyutin. We then show how a basic set of gauge invariant fields in the embedded model can be identified with the fundamental fields in the proca model as well as with the observables in the Stückelberg model or in the model involving the interaction of an abelian 2-form field with the Maxwell field. The connection of these models with the massive KalbRamond model is also elucidated within a path integral approach.
\end{abstract}


The generalised canonical formalism of Batalin, Fradkin and Tyutin (BFT) [1, 2] is a powerful approach to study the connection among different theories, in particular it has been effectively used [3, [1] to convert second class systems into true (i.e., first class) gauge theories by extending the phase space of the original theory. The partition function is then constructed which reduces in a definite (unitary) gauge to the partition function of the starting theory. It is also possible to compute the partition function for other choices of gauge. By virtue of the Fradkin-Vilkovisky [5] theorem, the theories corresponding to these partition functions (obtained by different gauge fixings) are all equivalent. The basic problem in this approach is to make a judicious choice of gauge that would yield some physically interesting theory. Indeed, as is quite well known, the choice of a viable gauge is a rather tricky and subtle issue [6]. Apart from this there is an arbitrariness in the manner in which the phase space can be extended. For example, by a straightforward (conventional) extension of the phase space of the proca model one can obtain, in FaddeevPopov like gauges the Stückelberg-embedded form of this model [4]. If, on the contrary, one adopts an alternative way of enlargement [7] whereby antisymmetric tensor fields are introduced, the Stückelberg scalar is replaced by 2-form gauge field. Consequently an equivalence between apparently distinct models may be achieved since these have a common origin.

The purpose of this paper is to systematically develop the canonical formalism such that the above mentioned ambiguities related to either gauge fixing or the embedding procedure are completely avoided. We present our ideas in the context of Proca model which has received considerable attention [4, 7]. The general ideas of BFT will be used to convert the second class constraints and Hamiltonian of the Proca model into their corresponding first class forms, by extending the phase space. It is then shown how by performing an inverse Legendre transformation in this extended space, the Stückelberg embedded Lagrangian of the Proca model emerges naturally. Going back to the extended phase space, we show that the gauge invariant fields in this space are equivalent to the fundamental fields in the Proca model. They satisfy the same algebra and have identical equations of motion. Furthermore, the involutive Hamiltonian can be expressed in terms of gauge invariant fields, modulo a term proportional to the generator of gauge transformation. This signalises the existence of an underlying gauge theory. One is then led in a natural way to the connection between the proca model and the gauge invariant sector of a model involving a massless 2-form gauge 
field interacting with a Maxwell field. It is important to point out that gauge fixing is not used at any stage of the analysis. Neither do we resort to different embedding procedures to obtain our results. We conclude by discussing a purely (configuration space) path integral approach which also does not involve any gauge fixing.

The Lagrangian for the Proca model is given by,

$$
\mathcal{L}=-\frac{1}{4} F_{\mu \nu} F^{\mu \nu}+\frac{m^{2}}{2} A_{\mu} A^{\mu}
$$

and leads to the field equations,

$$
\partial_{\mu} F^{\mu \nu}=-m^{2} A^{\nu}
$$

It describes a purely second class system with the constraints,

$$
\begin{aligned}
\Omega_{0} & =\Pi_{0} \approx 0 \\
\Omega & =\partial_{i} \Pi^{i}+m^{2} A_{0} \approx 0
\end{aligned}
$$

and the canonical Hamiltonian,

$$
H_{c}=\int d^{4} x\left[\frac{1}{2} \Pi_{i} \Pi_{i}+\frac{1}{4} F_{i j} F_{i j}-\frac{m^{2}}{2} A_{\mu} A^{\mu}-A_{0} \partial_{i} \Pi^{i}\right]
$$

where $\Pi_{i}=-F_{0 i}$ is the momentum conjugate to $A^{i}$.

We next convert the second class system into first class by adopting the basic ideas of BFT [1, 2]. The original phase space is enlarged by introducing a canonical pair of fields $\theta$ and $\Pi_{\theta}$. Then a new set of first class constraints can be defined in this extended space,

$$
\begin{aligned}
\Omega_{0}^{\prime} & =\Omega_{0}+m^{2} \theta \\
\Omega^{\prime} & =\Omega+\Pi_{\theta}
\end{aligned}
$$

which are strongly involutive. It is easy to verify that a Hamiltonian which is in involution with $\Omega_{0}^{\prime}$ and $\Omega^{\prime}$ is given by,

$$
H^{\prime}=H_{c}+\int\left(\frac{\Pi_{\theta}^{2}}{2 m^{2}}-\frac{m^{2}}{2} \partial_{i} \theta \partial^{i} \theta+m^{2} \theta \partial_{i} A^{i}\right)
$$


which satisfies the involutive Poisson algebra,

$$
\begin{aligned}
& \left\{\Omega_{0}^{\prime}(x), H^{\prime}\right\}=\Omega^{\prime}(x) \\
& \left\{\Omega^{\prime}(x), H^{\prime}\right\}=0 .
\end{aligned}
$$

The first class constraints $\Omega_{0}^{\prime}$ and $\Omega^{\prime}$ are the generators of gauge transformations,

$$
\begin{aligned}
\left\{A_{0}, G\left[\gamma_{0}\right]\right\} & =\gamma_{0} \\
\left\{A_{i}, G[\gamma]\right\} & =-\partial_{i} \gamma \\
\{\theta, G[\gamma]\} & =\gamma \\
\left\{\Pi_{\theta}, G\left[\gamma_{0}\right]\right\} & =-m^{2} \gamma_{0}
\end{aligned}
$$

where,

$$
\begin{aligned}
G\left[\gamma_{0}\right] & =\int d^{4} x \gamma_{0}(x) \Omega_{0}^{\prime}(x) \\
G[\gamma] & =\int d^{4} x \gamma(x) \Omega^{\prime}(x) .
\end{aligned}
$$

First we show how to obtain the Stückelberg form [4] by performing an inverse Legendre tranformation,

$$
\mathcal{L}^{\prime}=\Pi_{0} \dot{A}_{0}+\Pi_{i} \dot{A}^{i}+\Pi_{\theta} \dot{\theta}-H^{\prime}
$$

The momentum $\Pi_{0}$ is easily eliminated by using the constraint $\Omega_{0}^{\prime}$. The other two momenta $\Pi_{i}$ and $\Pi_{\theta}$ are eliminated by using Hamilton's equations of motion,

$$
\begin{aligned}
\dot{A}_{i}=\left\{A_{i}, H^{\prime}\right\} & =-\Pi_{i}+\partial_{i} A_{0} \\
\dot{\theta}=\left\{\theta, H^{\prime}\right\} & =\frac{\Pi_{\theta}}{m^{2}}
\end{aligned}
$$

and we find,

$$
\mathcal{L}^{\prime}=-\frac{1}{4} F_{\mu \nu} F^{\mu \nu}+\frac{1}{2} m^{2} A_{\mu} A^{\mu}+\frac{1}{2} m^{2} \partial_{\mu} \theta \partial^{\mu} \theta-m^{2} \theta \partial_{\mu} A^{\mu}
$$

which, upto a boundary term, reproduces the familiar Stückelberg structure [曰] with $\theta$ playing the role of the Stückelberg scalar [5],

$$
\mathcal{L}^{\prime}=-\frac{1}{4} F_{\mu \nu} F^{\mu \nu}+\frac{m^{2}}{2}\left(A_{\mu}+\partial_{\mu} \theta\right)\left(A^{\mu}+\partial^{\mu} \theta\right)
$$


We next show how the Proca model gets related to the abelian 2-form gauge field. It follows from (11) to (14) that the following combinations of fields,

$$
\begin{aligned}
F_{0} & =A_{0}+\frac{\Pi_{\theta}}{m^{2}} \\
F_{i} & =A_{i}+\partial_{i} \theta
\end{aligned}
$$

is gauge invariant and satisfy the algebra,

$$
\begin{aligned}
& \left\{F_{0}(x), F_{0}(y)\right\}=0 \\
& \left\{F_{0}(x), F_{i}(y)\right\}=\frac{1}{m^{2}} \partial_{i} \delta(\vec{x}-\vec{y}) \\
& \left\{F_{i}(x), F_{j}(x)\right\}=0
\end{aligned}
$$

The above algebra is precisely identical to the Dirac algebra [4] of the Proca fields in which case the constraint $\Omega_{0}, \Omega(3)$ are strongly implemented. Moreover, the $F_{\mu}$-fields satisfy the equation of motion,

$$
\partial_{\mu} G^{\mu \nu}=-m^{2} F^{\nu}+g^{0 \nu} \Omega^{\prime}
$$

obtained from the involutive Hamiltonian (8) and $G_{\mu \nu}=\partial_{\mu} F_{\nu}-\partial_{\nu} F_{\mu}$. Thus, modulo a term proportional to the first class constraint $\Omega^{\prime}$, the equations of motion (27) are identical to (2). We therefore conclude that the $F_{\mu}$ fields in the embedded (Stückelberg) version play the role of the fundamental fields $A_{\mu}$ in the Proca model. The next step is to express the involutive Hamiltonian (8) in terms of the $F_{\mu}$ fields,

$$
H^{\prime}=\int d^{3} x\left[\frac{1}{2} \Pi_{i}^{2}+\frac{1}{4} F_{i j}^{2}+\frac{m^{2}}{2}\left(F_{0}^{2}+F_{i}^{2}\right)\right]-\int d^{3} x A_{0}\left(\partial_{i} \Pi^{i}+m^{2} F_{0}\right)
$$

Observe that all reference to the original canonical pair $\left(\theta, \Pi_{\theta}\right)$ has been eliminated from the involutive Hamiltonian in favour of the $F_{\mu}$ fields. Furthermore, (28) is written in terms of gauge invariant fields with $A_{0}$ playing the role of the Lagrange multiplier associated with the generator of gauge transformations. It is clear, therefore, that this suggests an alternative way of exposing the underlying gauge symmetry through the pure Maxwell term (since this part is exactly reproduced in (28)) plus something which interacts with it. The structure of this 'remainder'can be recognised by realising that 
$F_{\mu}$ being gauge invariant and divergenceless (which follows from (27) and (10)) allows the introduction of an abelian 2-form gauge field $B_{\mu \nu}$ by,

$$
\begin{gathered}
F_{\mu}=\frac{1}{2} \epsilon_{\mu \nu \alpha \beta} \partial^{\nu} B^{\alpha \beta}=\frac{1}{6} \epsilon_{\mu \nu \alpha \beta} G^{\nu \alpha \beta} \\
G_{\nu \alpha \beta}=\partial_{[\nu} B_{\alpha \beta]}
\end{gathered}
$$

This is similar in spirit to the analysis in $2+1$ dimensions where the Hopf term could thereby be introduced in the nonlinear sigma model [9] or the equivalence between the Maxwell-Chern-Simons theory and a self dual model established [10]. The involutive Hamiltonian (28) may now be expressed as,

$$
\begin{aligned}
H^{\prime} & =\int\left[\frac{1}{2} \Pi_{i}^{2}+\frac{1}{4} F_{i j}^{2}-\frac{m^{2}}{12} G_{i j k} G^{i j k}+\frac{m^{2}}{4} G_{0 j k} G^{0 j k}\right] \\
& -\int A_{0}\left[\partial_{i} \Pi^{i}+\frac{m^{2}}{2} \epsilon_{i j k} \partial^{i} B^{j k}\right]
\end{aligned}
$$

One immediately recognises that Gauss operator associated with the Lagrange multiplier $A_{0}$ as that which occurs in the theory of an abelian 2-form field interacting with the Maxwell field and whose dynamics is governed by the Lagrangian density [7, 11],

$$
\tilde{\mathcal{L}}=-\frac{1}{4} F_{\mu \nu}^{2}-\frac{m^{2}}{6} \epsilon_{\mu \nu \rho \sigma} A^{\mu} G^{\nu \rho \sigma}+\frac{m^{2}}{12} G_{\mu \nu \rho} G^{\mu \nu \rho}
$$

It takes only a slight effort to show that the complete involutive Hamiltonian (31) follows from (32). The canonical momenta obtainable from (32) are given by,

$$
\begin{array}{rc}
\Pi_{0}=0 ; & \Pi_{0 i}=0 \\
\Pi_{i}=-F_{0 i} ; & \Pi_{i j}=m^{2}\left(\epsilon_{i j k} A^{k}+G_{0 i j}\right)
\end{array}
$$

and the canonical Hamiltonian is

$$
\begin{aligned}
\tilde{H} & =\int d^{3} x\left[\Pi_{i} \dot{A}^{i}+\frac{1}{2} \Pi_{i j} \dot{B}^{i j}-\tilde{\mathcal{L}}\right] \\
& =\int d^{3} x\left[\frac{1}{2} \Pi_{i}^{2}+\frac{m^{2}}{2} A_{i}^{2}+\frac{1}{4} F_{i j}^{2}+\frac{1}{4 m^{2}} \Pi_{i j}^{2}+\frac{1}{2} \epsilon_{i j k} A_{i} \Pi_{j k}\right. \\
& \left.+\frac{m^{2}}{12} G_{i j k}^{2}-A_{0}\left(\partial_{i} \Pi^{i}+\frac{m^{2}}{2} \epsilon_{i j k} \partial^{i} B^{j k}\right)+B_{0 j} \partial_{i} \Pi_{i j}\right]
\end{aligned}
$$


Apart from the primary constraints (33) there are two secondary constraints,

$$
\begin{aligned}
\partial_{i} \Pi^{i}+\frac{m^{2}}{2} \epsilon_{i j k} \partial^{i} B^{j k} & \approx 0 \\
\partial_{i} \Pi_{i j} & \approx 0
\end{aligned}
$$

As expected, all these constraints are first class. Using the definition for the canonical momenta $\Pi_{i j}$ (34), it is easy to show that (31) maps on to (35) modulo a term proportional to the first class constraint $\partial_{i} \Pi_{i j}=0$. Since the physical states are annihilated by such constraints the equivalence between (31) and (35) in the gauge invariant sector is established. We therefore find out that the Proca model is alternatively mapped on to the gauge invariant sector of models defined by either the Stückelberg Lagrangian (21) or the Maxwell-Kalb-Ramond (MKR) Lagrangian (35), both of which are derived within a unified canonical framework.

Furthermore the correspondence among the basic fields in these models is given by,

$$
\begin{gathered}
\left(A_{0}\right)_{\text {Proca }} \leftrightarrow\left(A_{0}+\frac{\Pi_{\theta}}{m^{2}}\right)_{\text {Stückelberg form }} \leftrightarrow\left(\frac{1}{6} \epsilon_{i j k} G^{i j k}\right)_{\mathrm{MKR}} \\
\left(A_{i}\right)_{\text {Proca }} \leftrightarrow\left(A_{i}+\partial_{i} \theta\right) \text { Stückelberg form } \leftrightarrow\left(-\frac{1}{2} \epsilon_{i j k} G^{0 j k}\right)_{\mathrm{MKR}}
\end{gathered}
$$

and are the analouges of the corresponding mappings obtained by one of us [10] in $2+1$ dimensional case.

The Proca model or its equivalent formulations describe the propagation of a single massive mode. It is known [11] that this is also true for a model described by a free massive abelian 2-form potential - the Kalb-Ramond (KR) model whose Lagrangian is given by,

$$
\mathcal{L}_{K R}=\frac{1}{12} G_{\mu \nu \rho} G^{\mu \nu \rho}-\frac{1}{4} B_{\mu \nu} B^{\mu \nu}
$$

We shall conclude this paper by explicitly revealing the connection of (40) with the Proca model (11) and thereby with the other alternative formulations (21) and (35). To this effect consider the following master Lagrangian,

$$
\mathcal{L}_{M}=\frac{m^{2}}{2} A_{\mu} A^{\mu}-\frac{m^{2}}{4} B_{\mu \nu} B^{\mu \nu}+\frac{m}{6} \epsilon_{\alpha \mu \nu \rho} A^{\alpha} G^{\mu \nu \rho}
$$


Master Lagrangians, incidentally, have been exploited earlier [12] to discuss the equivalence among various theories but these were usually confined to $2+1$ dimensions [13]. The partition function corresponding to (41), in the presence of external sources $J_{\mu}, K_{\mu}$, is given by,

$$
Z_{M}=\int \mathcal{D} A_{\mu} \mathcal{D} B_{\mu \nu} \exp i \int d^{4} x\left(\mathcal{L}_{M}+J_{\mu} A^{\mu}+\frac{K_{\mu}}{6} \epsilon_{\mu \alpha \beta \sigma} G^{\alpha \beta \sigma}\right)
$$

Performing the gaussian integration over the abelian 2-form field $B_{\mu \nu}$ yields,

$$
Z_{M}=\int \mathcal{D} A_{\mu} \exp i \int d^{4} x\left(\frac{m^{2}}{2} A_{\mu} A^{\mu}-\frac{1}{4} F_{\mu \nu}^{2}+J_{\mu} A^{\mu}+\frac{1}{m^{2}} K_{\beta} \partial_{\alpha} F^{\alpha \beta}\right)
$$

where a nonpropagating contact term has been dropped. In the absence of sources (43) is exactly the partition function for the proca model [1].

Alternatively, doing the $A_{\mu}$-integration in (42) leads to,

$$
\begin{aligned}
Z_{M} & =\int \mathcal{D} B_{\mu \nu} \exp i \int d^{4} x\left(\frac{1}{12} G_{\mu \nu \rho} G^{\mu \nu \rho}-\frac{m^{2}}{4} B_{\mu \nu} B^{\mu \nu}\right. \\
& \left.+\frac{1}{6} \epsilon_{\mu \alpha \beta \sigma} G^{\alpha \beta \sigma}\left(J^{\mu}+K^{\mu}\right)\right)
\end{aligned}
$$

where, once again, a nonpropagating contact term has been ignored. In the absence of sources (44) is the partition function for the Kalb-Ramond model (40). Since (43) and (44) were derived from a common origin, it establishes their duality. Furthermore, comparing the source terms proportional to $J_{\mu}$, $K_{\mu}$, the following identifications are obtained,

$$
\left(A_{\mu}\right)_{\text {Proca }} \leftrightarrow\left(-\frac{1}{m^{2}} \partial^{\alpha} F_{\alpha \mu}\right)_{\text {Proca }} \leftrightarrow\left(-\frac{1}{6 m} \epsilon_{\mu \alpha \beta \sigma} G^{\alpha \beta \sigma}\right)_{\mathrm{KR}}
$$

It is straightforward to reproduce the equations of motion of either the Proca field or the Kalb-Ramond field from the above correspondence. This correspondence can also be used, in conjunction with (38), (39) to relate the basic fields in the Stückelberg and MKR versions with those in the KR model.

To conclude, we have employed the general notions of Batalin, Fradkin and Tyutin 11, 2] to obtain a deeper insight into the connection between the fields of the Proca model and those in the corresponding Stückelberg embedded version or in a model involving the interaction of a massless abelian 
2-form field with the Maxwell field (Maxwell-Kalb-Ramond model). A significant aspect of this work was to provide a unique enlargement technique of the phase space of the original Proca model which led, in a systematic and natural way, to the gauge invariant sector of either the Stückelberg type model or the MKR model. The use of different enlargement prescriptions, as advocated in the literature [1, 2, [7], can therefore be avoided. Moreover, since gauge fixing was not necessary at any stage of the computations, subtleties and ambiguities involved in such a procedure were eliminated. We also furnished a path integral formulation, whereby starting from a master Lagrangian the duality between the Proca model and the massive Kalb Ramond model was established. An identification between the fields in the respective models was also obtained. The master Lagrangian was gauge fixed from the begining so that the problem of gauge fixing was once again bypassed. Although the computations were presented in $3+1$ dimensions, it is easy to extend these to arbitrary $d+1(d \geq 3)$ dimensions. The Proca field in that case would be connected to a $d-1$-form abelian field. The $d=2$ example, incidentally, is special since it admits the Chern Simons 3-form and has been discussed extensively in the literature [12, 14]. 


\section{References}

[1] I. A. Batalin and E. S. Fradkin, Nucl. Phys. B279 (1987) 514.

[2] I. A. Batalin and I. V. Tyutin, Int. J. Mod. Phys. A6 (1991) 3255.

[3] Y. W. Kim, S. K. Kim, W. T. Kim, Y. J. Park, K. Y. Kim and Y. Kim, Phys. Rev. D46 (1992) 4574

R. Banerjee, Phys. Rev. D48 (1993) R5467

R. Banerjee, H. J. Rothe and K. D. Rothe, Phys. Rev. D49 (1994) 5436;

Nucl. Phys. B426 (1994) 129

N. Banerjee, R. Banerjee and S. Ghosh, Nucl. Phys. B427 (1994) 257;

Phys. Rev. D49 (1994) 1996.

[4] N. Banerjee, R. Banerjee and S. Ghosh, Anns. Phys. 241 (1995) 237

O. Daye, Phys. Lett. B210 (1988) 147

T. Fujiwara, Y. Igarashi and J. Kubo, Nucl. Phys. B341 (1990) 695.

[5] E. S. Fradkin and G. A. Vilkovisky, Phys. Lett. B55 (1975) 224

M. Henneaux, Phys. Rep. C126 (1985) 1.

[6] M. Henneaux and C. Teitelboim, 'Quantisation of gauge systems' Princeton University Press, Princeton,1992.

[7] H. Sawayanagi, Mod. Phys. Lett. A10 (1995) 813.

[8] E. C. G. Stückelberg, Helv. Phys. Act.30 (1957) 209.

[9] F. Wilckek and A. Zee, Phys. Rev. Lett. 51 (1983) 2250.

[10] R. Banerjee and H. J. Rothe, Nucl. Phys. B 447 (1995) 183.

[11] E. Cremmer and J. Scherk, Nucl. Phys. B72 (1974) 117

A. Lahiri, Mod. Phys. Lett. A8 (1993) 2403

T. J. Allen, M. J. Bowick and A. Lahiri, Mod. Phys. Lett. A6 (1991) 559 .

[12] S. Deser and R. Jackiw, Phys. Lett. B139 (1984) 371

E. Fradkin and F. A. Scaposnik, Phys. Lett. B338 (1994) 253. 
[13] For a discussion in higher dimensions, see, R. Banerjee, Duality and bosonisation in arbitrary dimensions, hepth/9509133 ; K. Kondo, Bosonisation and duality of massive Thirring model, hepth/9502100.

[14] P. K. Townsend, K. Pilch and P. van Nieuwenhuizen, Phys. Lett. B136 (1984) 38; ibid B137 (1984) 443(addendum). 